\title{
Quantum self-consistent approach to the charge gap of the quasi-one-dimensional organic conductors
}

\author{
Xuefan Jiang ${ }^{\mathrm{a}, \mathrm{b}, *}$, G.Y. Guo ${ }^{\mathrm{b}}$ \\ ${ }^{a}$ Department of Physics, Changshu Institute of Technology, Changshu, 215500 Jiangsu, China \\ ${ }^{\mathrm{b}}$ Department of Physics, National Taiwan University, Taipei 106, Taiwan
}

Received 5 May 2003; received in revised form 4 September 2003; accepted 12 November 2003 by S.G. Louie

\begin{abstract}
We apply an extended quantum self-consistent method, in which quantum fluctuations are taken into account, to the bosonization Hamiltonian to investigate analytically the charge gap in the quasi-one-dimensional (1D) organic conductors at quarter-filling described by the 1D Hubbard model with dimerization and alternate potential on site. It is shown that either dimerization or alternate potential gives rise to the enhancement of the charge gap. Our results are compared with those of the numerical and the other analytical theories. Our results are also consistent with the experimental data of the actual organic materials when the effect of nearest-neighbor Coulomb interaction is taken into account.
\end{abstract}

(C) 2003 Elsevier Ltd. All rights reserved.

PACS: 71.30. + h; 71.20.Rv; 71.45.Lr

Keywords: A. Organic crystals; A. Superconductors; D. Electronic state; D. Electronic transport

Quasi-one-dimensional (1D) organic conductors have been a subject of great interest because of a variety of electronic states with spatially inhomogeneous charge, spin, and lattice structures. A typical example is the family of the Bechgaard salts (TMTSF) $)_{2} \mathrm{X}$ and (TMTTF) $)_{2} \mathrm{X}[1]$, with $\mathrm{X}=\mathrm{PF}_{6}, \mathrm{Br}$, $\mathrm{ClO}_{4}$ and $\mathrm{ReO}_{4}$, etc. These materials have a 3/4-filled electron (i.e., quarter-filled hole) band due to a charge transfer from two organic molecules to one counterion $\mathrm{X}$. Since the transfer integral is slightly dimerized along the chain axis, the system becomes effectively half-filling.

Experimentally, the states above the transition temperature, $T_{\mathrm{tr}}$, are unusual, indicating the behavior of a charge gap, $\Delta_{\rho}$. For $(\mathrm{TMTTF})_{2} \mathrm{X}$ salts [1], the resistivity has a minimum at a temperature $T_{\rho}$, which is in the range $\sim 100-$ $300 \mathrm{~K}$, corresponding to the gap, $\Delta_{\rho} \simeq \pi T_{\rho}$. Below $T_{\rho}$, but above $T_{\text {tr }}$, the resistivity increases with decreasing temperature. In contrast, the (TMTSF) $)_{2} \mathrm{X}$ analogs display a metallic

\footnotetext{
* Corresponding author. Address: Department of Physics, Changshu College, Changshu 215500, Changshu Jiangsu, China. Tel.: + 86-512-527-062-47; fax: + 86-512-527-734-86.

E-mail address: jxfjxf@jlonline.com (X. Jiang).
}

character. The resistivity of (TMTSF) $)_{2} \mathrm{X}$ salts decreases monotonically with decreasing temperature for $T>T_{\mathrm{tr}}$. However, in both groups of salts, no anomaly is found in the magnetic susceptibility. The nearly temperature-independent magnetic susceptibility [2] gives strong evidence for a gapless spin excitation spectrum. In particularly, recent optical and photoemission experiments [3] have demonstrated the charge gap for both TMTTF and TMTSF salts. It concludes that (TMTTF) ${ }_{2} \mathrm{X}$ exhibit a Mott-Hubbard charge localization below $T_{\rho}$, while (TMTSF) $)_{2} \mathrm{X}$ are good metals [4]. Theory [4] predicts that the electron-electron interactions together with Umklapp scattering result in a charge gap for these conductors. The hopping integral of the dimerization and the $4 k_{\mathrm{F}}$-periodic alternating potential, where $k_{\mathrm{F}}$ is the Fermi wave vector of quarter-filling, become two scattering mechanisms, which give rise to the charge gap, as found separately in TMTSF and TMTTF salts [5]. Furthermore, the organic alloys such as $\left[(\mathrm{TMTSF})_{1-x}(\mathrm{TMTTF})_{x}\right]_{2} \mathrm{ReO}_{4}$ around $x \sim 0.5$ exhibit an enhanced charge localization [6], which is attributed to a $4 k_{\mathrm{F}}$ order of charge on the site due to the alternate order of TMTSF and TMTTF molecules. As a minimal model in this 
case, a dimerized Hubbard model with an alternating atomic on-site energies would be appropriate to describe the properties of these transfer charges.

Recently, extensive theoretical studies have been carried out [7-10]. It has generally been accepted that the electronic properties of quasi 1D organic conductors is described by the dimerized Hubbard model at quarter filling, which is a natural extension of the extended Hubbard model to include the electron-lattice coupling. A detailed study of the charge gap behavior for the 1D quarter-filled Hubbard model with the dimerized hopping integrals or the alternating potential has been examined by Penc and Mila [7], in which the analytical results obtained in various limits and numerical results obtained by exact diagonalization of small clusters have been shown. Moreover, the densitymatrix renormalization-group (DMRG) technique [8] has also been used to study the charge gap in the 1D dimerized Hubbard model at quarter filling. At the same time, the charge gap in the quarter-filled organic conductor with dimerization and/or alternating potential was studied by applying the renormalization group (RG) method [9] to the bosonized 1D Hamiltonian to be consistent qualitatively with that from the numerical calculations $[7,8]$. We note that the values of the charge gap in the weak coupling regime from the RG technique [9] are in agreement with those obtained by the DMRG [8], however, in the large and intermediate coupling regimes where actual materials are located, the RG technique yields much larger values in comparison with the DMRG results [8]. Existing theoretical studies, mainly numerical ones for the charge gap, have failed to compare with the experimental data in both $(\text { TMTSF })_{2} \mathrm{X}$ and $(\mathrm{TMTTF})_{2} \mathrm{X}$. They are generally much smaller than those of experiments [3], although could be enhanced by taking into account the effect of the nearestneighbor Coulomb repulsion. In this paper, we start from the bosonization results of Ref. [9], but apply a so-called quantum self-consistent theory, which was proposed [11] to deal with the 1D quantum sine-Gordon (SG) model, to the bosonization Hamiltonian to examine the charge gap of the 1D organic conductors at quarter-filling. We wish to not only present an analytical approach but also obtain a more accurate theoretical estimation of the charge gap compared with the results of the numerical calculations in the organic conductors and alloys.

We herewith consider a 1D Hamiltonian given by

$$
\begin{aligned}
H= & -\sum_{i, \sigma}\left[t+(-1)^{i} t_{\mathrm{d}}\right]\left(C_{i, \sigma}^{+} C_{i+1, \sigma}+\text { h.c. }\right)+W_{0} \sum_{i, \sigma} \\
& \left.\times(-1)^{i} n_{i, \sigma}+U \sum_{i} n_{i, \uparrow} n_{i, \downarrow}\right) .
\end{aligned}
$$

where $C_{i, \sigma}^{+}\left(C_{i, \sigma}\right)$ is the creation (annihilation) operator of an electron with spin $\sigma\left(\uparrow\right.$ or $\downarrow$ ) at site $i$, and $n_{i}\left(=n_{i, \uparrow}+n_{i, \downarrow}\right)$ is the electron-number operator. There are two kinds of the hopping integrals for the nearest neighbor sites along the 1D chain, $t_{1}\left(=t+t_{\mathrm{d}}\right)$ and $t_{2}\left(=t-t_{\mathrm{d}}\right)$ with $t_{\mathrm{d}} \geq 0$, due to the lattice dimerization. The quantities $W_{0}$ and $U$ are the amplitudes of the alternating potential and the on-site electron-electron Coulomb repulsive interaction, respectively. Since a gap appears at $k= \pm \pi / 2 a$, where $a$ is the lattice constant, the first Brillouin zone reduces to half so that the system can be regarded effectively as half-filling with Fermi momentum, $k_{\mathrm{F}}=\pi / 4 a$.

The version of Eq. (1) can usually be treated analytically by introducing the bosonized phase variables under the continuous limit approximation. The effective Hamiltonian consists of two parts of the charge degree and the spin degree of freedom. As a starting point, we use the bosonized effective Hamiltonian from Ref. [9] in which the charge part is expressed in terms of bosonization with the phase variable as

$$
\begin{gathered}
H_{\rho}=\frac{v_{\rho}}{4 \pi} \int \mathrm{d} x\left[K_{\rho}\left(\pi \Pi_{\rho}\right)^{2}+\frac{1}{K_{\rho}}\left(\partial_{x} \phi_{\rho}\right)^{2}\right]-\frac{\lambda_{1}}{2 \pi^{2} \alpha^{2}} \int \\
\mathrm{d} x \sin \left(2 \phi_{\rho}\right)-\frac{\lambda_{2}}{2 \pi^{2} \alpha^{2}} \int \mathrm{d} x \cos \left(2 \phi_{\rho}\right),
\end{gathered}
$$

where $\Pi_{\rho}$ is the momentum density field conjugate to field operator $\phi_{\rho}$, the commutation relation, $\left[\phi_{\rho}(x), \Pi_{\rho}\left(x^{\prime}\right)\right]=$ $\mathrm{i} \delta\left(x-x^{\prime}\right)$, holds. $K_{\rho}=\left[2 \pi v_{\mathrm{F}} /\left(2 \pi v_{\mathrm{F}}+2 g\right)\right]^{1 / 2}$ and $v_{\rho}=$ $v_{\mathrm{F}}\left[1-g /\left(\pi v_{\mathrm{F}}\right)\right]^{1 / 2} \quad$ with $g=U a\left[1+W_{0}^{2} /\left(t_{1}^{2}+t_{2}^{2}+W_{0}^{2}\right)\right]$ and $v_{\mathrm{F}}=2 t_{1} t_{2} a /\left(t_{1}^{2}+t_{2}^{2}+W_{0}^{2}\right)^{1 / 2}$. The quantity $\alpha\left(\sim 1 / k_{\mathrm{F}}\right)$ is a cutoff of the order of the lattice constant. The coefficients, $\lambda_{1,2}$, are related to the various parameters as $\lambda_{1}=2 U a\left[\left(t_{1}-\right.\right.$ $\left.\left.t_{2}\right) /\left(t_{1}+t_{2}\right)\right] /\left[1+\left(t_{1}-t_{2}\right)^{2} /\left(t_{1}+t_{2}\right)^{2}\right] \quad\left[1+W_{0}^{2} /\left(t_{1}^{2}+t_{2}^{2}+\right.\right.$ $\left.\left.W_{0}^{2}\right)\right] \quad$ and $\quad \lambda_{2}=2 U a\left[W_{0} / \sqrt{t_{1}^{2}+t_{2}^{2}+W_{0}^{2}}\right]\left\{1-2\left(t_{1}-\right.\right.$ $\left.\left.t_{2}\right)^{2} /\left(t_{1}+t_{2}\right)^{2} /\left[1+\left(t_{1}-t_{2}\right)^{2} /\left(t_{1}+t_{2}\right)^{2}\right]\right\}$. We set $a=1$ below.

In order to solve Eq. (2), we note that there are several effective approximations [11-13] to treat the 1D SG model, among which a so-called quantum self-consistent theory [12] can effectively treat the infrared divergence and obtain self-consistently the energy gap in the spectrum. It is worth to notice that Eq. (2) reduces to the standard SG model [14] when $\lambda_{1}=0$ or $\lambda_{2}=0$. In Eq. (2) the classical effective potential occurs at a stable point at $\phi_{\rho}=0$ (or $\phi_{\rho}=\pi / 4$ ) as $\lambda_{1}=0$ (or $\lambda_{2}=0$ ). In contrast to the standard SG model, it is necessary to determine the stable point of the phase variable defined as $\phi_{0}$, corresponding to the minimum of the classical effective potential. Thus, we can make the perturbational expansion of the nonlinear terms in Eq. (2) around $\phi_{0}$. When quantum fluctuation is taken into account the classical effective potential can be regarded as to be renormalized by quantum fluctuation. In order to treat the classical and quantum parts simultaneously, we divide the phase variable, $\phi_{\rho}$, into two parts: $\phi_{\rho}=\tilde{\phi}_{\rho}+\phi_{0}$, where $\tilde{\phi}_{\rho}$ stands for quantum fluctuation, $\phi_{0}$ being classical stable point, which will be self-consistently calculated, instead of $\phi_{0}=0$ in the standard SG model. Then the field $\tilde{\phi}_{\rho}$ and its canonical moment $\tilde{\Pi}_{\rho}$ can be expanded in moment space as

$\tilde{\phi}_{\rho}(x)=\sum_{k}(2|k|)^{-1 / 2} \exp \left(-\gamma_{k}\right) \exp (\mathrm{i} k x)\left(b_{k}+b_{-k}^{\dagger}\right)$, 
$\tilde{\Pi}_{\rho}(x)=-\mathrm{i} \sum_{k}(|k| / 2)^{1 / 2} \exp \left(\gamma_{k}\right) \exp (\mathrm{i} k x)\left(b_{k}-b_{-k}^{\dagger}\right)$.

where the summation over $k$ is performed in the range $0 \leq$ $|k| \leq \pi$. The above definitions are different from the original expression for the field variable $\tilde{\phi}_{\rho}(x)$ in terms of annihilation and creation operators, in which we have considered the infrared divergences induced by the massless scalar field with the help of the parameters $\gamma_{k}$ s. They will be determined by self-consistent results in the following, the operators $b_{k}$ and $b_{k}^{\dagger}$ satisfy the standard boson commutator. Inserting Eqs. (3) and (4) into the bosonized Hamiltonian Eq. (2) and normally ordering two nonlinear terms in $H_{\rho}$ and then expanding them to second order in terms of the bosonic operators we obtain

$H_{\rho}=H_{0}+H_{1}+H_{2}+H_{3}$,

where

$$
\begin{aligned}
& \begin{aligned}
H_{0}= & v_{\rho} \sum_{k} \frac{1}{2}|k| \cosh \left(2 \gamma_{k}\right)-\frac{\lambda_{1} \xi}{2 \pi^{2} \alpha^{2}} \sin \left(\sqrt{8 \pi K_{\rho}} \phi_{0}\right) \\
& -\frac{\lambda_{2} \xi}{2 \pi^{2} \alpha^{2}} \cos \left(\sqrt{8 \pi K_{\rho}} \phi_{0}\right), \\
H_{1}= & \sum_{k}\left\{v_{\rho}|k| \cosh \left(2 \gamma_{k}\right)+\frac{2 K_{\rho} \exp \left(-2 \gamma_{k}\right)}{\pi \alpha^{2}|k|}\right. \\
\times & {\left.\left[\lambda_{1} \xi \sin \left(\sqrt{8 \pi K_{\rho}} \phi_{0}\right)+\lambda_{2} \xi \cos \left(\sqrt{8 \pi K_{\rho}} \phi_{0}\right)\right]\right\} b_{k}^{+} b_{k}, }
\end{aligned}
\end{aligned}
$$

interaction term is taken only into account, although the formulation is different. Then the diagonalization of the Hamiltonian $H_{\rho}$ amounts to solve the following coupled equations

$$
\begin{aligned}
& \lambda_{1} \cos \left(\sqrt{8 \pi K_{\rho}} \phi_{0}\right)-\lambda_{2} \sin \left(\sqrt{8 \pi K_{\rho}} \phi_{0}\right)=0, \\
& \gamma_{k}=\frac{1}{4} \ln \left\{1+\frac{4 K_{\rho}}{\pi \alpha^{2} v_{\rho} k^{2}}\left[\lambda_{1} \xi \sin \left(\sqrt{8 \pi K_{\rho}} \phi_{0}\right)\right.\right. \\
& \left.\left.+\lambda_{2} \xi \cos \left(\sqrt{8 \pi K_{\rho}} \phi_{0}\right)\right]\right\} .
\end{aligned}
$$

Thus, $H_{\rho}$ becomes a massive free field

$$
H_{\rho}=\sum_{k} \omega_{k} b_{k}^{+} b_{k}+E_{0}
$$

where $E_{0}$ stands for an energy constant, $\omega_{k}$ is the elementary excitation spectrum and written as $\omega_{k}=\sqrt{v_{\rho}^{2} k^{2}+\Delta_{\rho}^{2}}, \Delta_{\rho}$ is the charge gap which is given by

$$
\begin{aligned}
\Delta_{\rho}^{2}= & 4 v_{\rho} K_{\rho} \xi\left[\lambda_{1} \sin \left(\sqrt{8 \pi K_{\rho}} \phi_{0}\right)\right. \\
& \left.+\lambda_{2} \cos \left(\sqrt{8 \pi K_{\rho}} \phi_{0}\right)\right] /\left(\pi \alpha^{2}\right) .
\end{aligned}
$$

Considering Eqs. (10) and (12), a self-consistent equation of $\xi$ can be driven as

$\xi^{1 / K_{\rho}-1}=\frac{4 K_{\rho} \lambda_{1} \sin \left(\sqrt{8 \pi K_{\rho}} \phi_{0}\right)+4 K_{\rho} \lambda_{2} \cos \left(\sqrt{8 \pi K_{\rho}} \phi_{0}\right)}{\left[\sqrt{\pi v_{\rho}}+\sqrt{\left.\pi v_{\rho}+4 K_{\rho} \lambda_{1} \xi \sin \left(\sqrt{8 \pi K_{\rho}} \phi_{0}\right)+4 K_{\rho} \lambda_{2} \xi \cos \left(\sqrt{8 \pi K_{\rho}} \phi_{0}\right)\right]^{2}}\right.}$.

$$
\begin{aligned}
H_{2}= & \int \mathrm{d} x \sum_{k} \frac{\exp \left(-\gamma_{k}\right)}{2 \pi^{2} \alpha^{2} \sqrt{2|k|}}\left[\lambda_{1} \xi \cos \left(\sqrt{8 \pi K_{\rho}} \phi_{0}\right)\right. \\
& \left.-\lambda_{2} \xi \sin \left(\sqrt{8 \pi K_{\rho}} \phi_{0}\right)\right]\left(b_{k} \mathrm{e}^{\mathrm{i} k x}+b_{k}^{+} \mathrm{e}^{-\mathrm{i} k x}\right), \\
H_{3}= & -\frac{1}{2} \sum_{k}\left\{v_{\rho}|k| \sinh \left(2 \gamma_{k}\right)-\frac{2 K_{\rho} \exp \left(-2 \gamma_{k}\right)}{\pi \alpha^{2}|k|}\right. \\
& \left.\times\left[\lambda_{1} \xi \sin \left(\sqrt{8 \pi K_{\rho}} \phi_{0}\right)+\lambda_{2} \xi\left(\sqrt{8 \pi K_{\rho}} \phi_{0}\right)\right]\right\} \\
& \times\left(b_{k}^{+} b_{-k}^{+}+b_{k} b_{-k}\right),
\end{aligned}
$$

with

$\xi=\exp \left(-2 \pi K_{\rho} \sum_{k} \frac{\mathrm{e}^{-2 \gamma_{k}}}{|k|}\right)$.

Note that above treatment is somewhat similar to the self-consistent harmonic approximation (SCHA) [11], in which the cosine term in the SG model is expanded up to second order term including field scale to obtain a solvable Hamiltonian. The present result turns out to be the same as that of SCHA if the second order
It should be pointed out that for Eq. (15), there is always a nonzero solution of $\xi$ because $K_{\rho} \leq 1$, corresponding to the stable point of energy [12]. This result provides that the 1D Hubbard system with the dimerized hopping integrals and/ or the alternating on-site potential is in the insulating state except for the noninteracting case $(U=0)$, since the charge gap is proportional to the parameter, $\xi$, as seen from Eq. (14).

In Fig. 1(a) and (b) we plot the charge gap as a function of the coupling for various values of $t_{2} / t_{1}$ at $W_{0}=0$ and of $W_{0} / t_{1}$ at $t_{2} / t_{1}=1$, respectively, in the units of $t_{1}$. We find that even if dimerization or alternating potential exists, the charge gap always vanishes at $U=0$. In the weak-coupling limit, $U \ll t_{1}, t_{2} ; W_{0}$, the charge gap is expected to increase exponentially with increasing $U / t_{1}$, which is in good agreement with those obtained by the previous numerical [7,8] and analytical [7,9] techniques. In the inset of Fig. 1(a) for the case of the large dimerization we can also see that the charge gap undergoes a crossover from the exponential behavior to the linear one with increasing $U / t_{1}$ from zero. In the large and intermediate $U$ regimes, our results are qualitatively consistent with but quantitatively somewhat larger than that from the numerical calculation $[7,8]$, while 

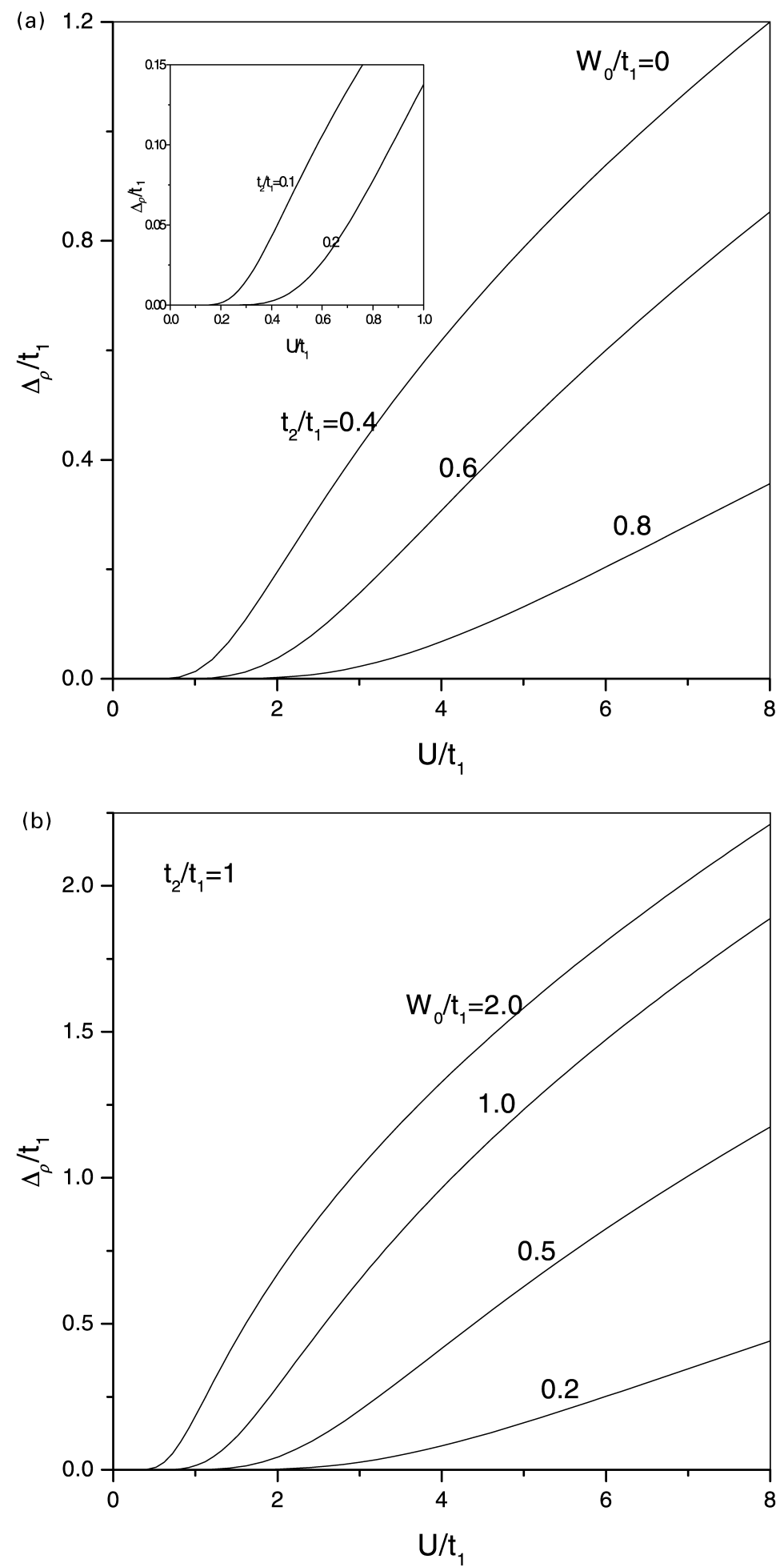

Fig. 1. The charge gap $\Delta_{\rho}$ as a function of the on-site Coulomb repulsion $U$ (a) for various values of $t_{2} / t_{1}$ at $W_{0}=0$ and (b) for various $W_{0} / t_{1}=1$, at $t_{2} / t_{1}=1$, in the units of $t_{1}$. 


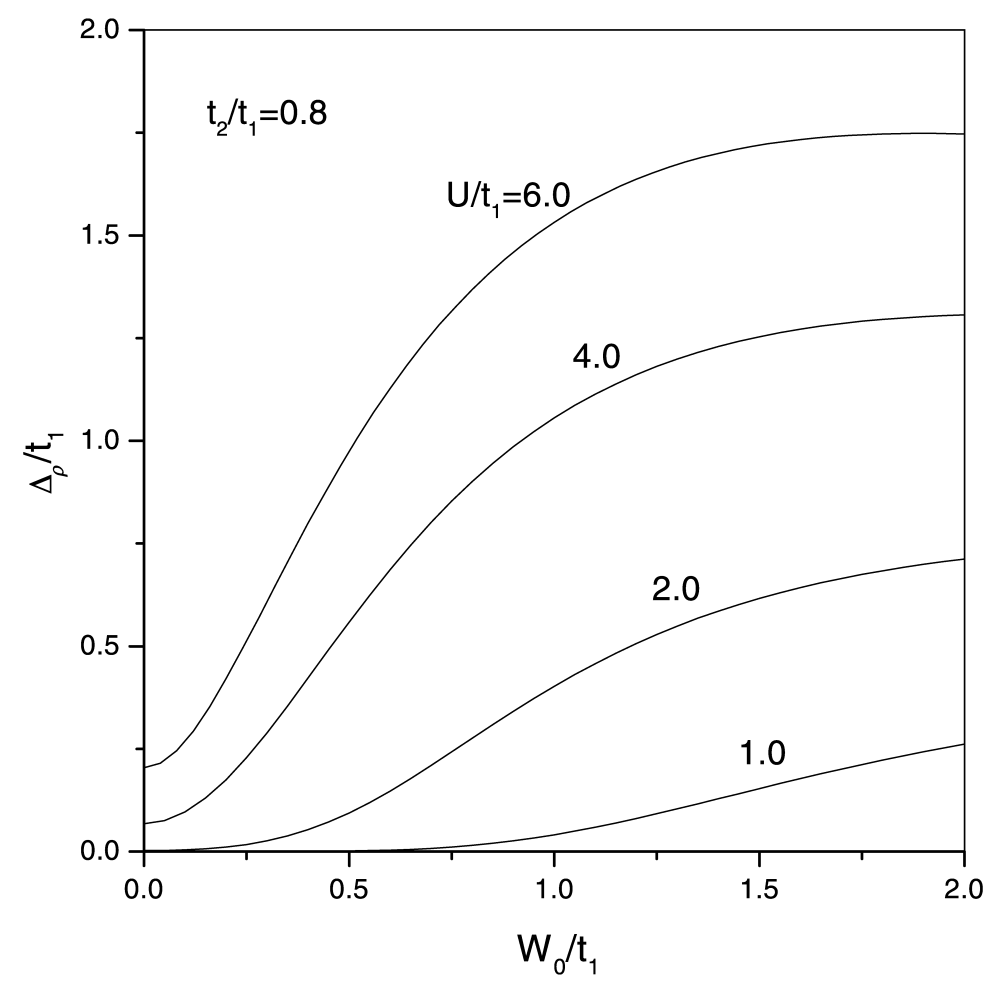

Fig. 2. The $W_{0} / t_{1}$ dependence of the reduced charge gap, $\Delta_{\rho} / t_{1}$, for $U / t_{1}=1.0,2.0,4.0$, and 6.0 at $t_{2} / t_{1}=0.8$.

somewhat smaller than those of the RG technique [9]. For example, the RG technique gives more than threefold larger values at $t_{2} / t_{1}=0.7$ and $U / t_{1}=7.0$ for $W_{0} / t_{1}=0$ in comparison with that of the DMRG technique [8], while our results gives about twofold larger.

In Fig. 2 the $W_{0} / t_{1}$ dependence of the charge gap, $\Delta_{\rho} / t_{1}$, is shown for some values of $U / t_{1}$ at $t_{2} / t_{1}=0.8$. It shows the enhancement of the charge gap with increasing alternating potential, indicating an enhanced charge localization due to the alternate order of TMTSF and TMTTF molecules in the organic alloys. The increase in $\Delta_{\rho}$ with $W_{0}$ is steep for the large or intermediate $U$ values, which represents the noticeable interplay between the alternating potential and the on-site Coulomb repulsion. However, the variation of $\Delta_{\rho}$ with $W_{0}$ becomes slower, like a saturation case, for $W_{0} / t_{1} \geq 1$.

Now, we focus on a brief comparison to experimental data of the charge gap in the, (TMTTF $)_{2} \mathrm{X},(\mathrm{TMTSF})_{2} \mathrm{X}$ and [(TMTSF $\left.)_{0.5}(\mathrm{TMTTF})_{0.5}\right]_{2} \mathrm{RO}_{4}$. From optical experiments on TMTTF and TMTSF salts, the charge gap is estimated to be $\Delta_{\rho} \simeq 100$ and $25 \mathrm{meV}$ for $(\mathrm{TMTTF})_{2} \mathrm{PF}_{6}$ and (TMTSF $)_{2} \mathrm{ClO}_{4}$ [3], respectively. We choose the parameter values as $t_{1}=137 \mathrm{meV}, \quad t_{2}=93 \mathrm{meV}$ and $U=959 \mathrm{meV}$ for (TMTTF $)_{2} \mathrm{PF}_{6}$ and $t_{1}=287 \mathrm{meV}, t_{2}=266 \mathrm{meV}$ and $U=1435 \mathrm{meV}$ for (TMTSF) ${ }_{2} \mathrm{ClO}_{4}[9,15,16]$. At $W_{0}=0$, the charge gaps obtained from our calculation are $\Delta_{\rho}=75.4$ and $6.21 \mathrm{meV}$ for (TMTTF) $)_{2} \mathrm{PF}_{6}$ and (TMTSF) $)_{2} \mathrm{ClO}_{4}$, respectively. The values of $\Delta_{\rho}$ are much smaller than those of experiments [3], but which can be enhanced by taking into account the effect of the nearest-neighbor Coulomb interaction, $V$, [8]. From the estimation of the DMRG method, the charge gap depends strongly on $V$ in Bechgaard salts. Comparing with the other theoretical results, which are listed in Table 1, our results are nearly twofold larger than that of the DMRG technique [8], while, substantially smaller than those from the RG method [9]. As an analytical approximation, in the present theory the quantum fluctuations are taken into account. In similar manner, we follow Ref. [9] to choose the parameters as $t_{1}=140 \mathrm{meV}, \quad t_{2}=100 \mathrm{meV}, \quad U=959 \mathrm{meV} \quad$ and $W_{0}=0 \mathrm{meV}$ for (TMTTF) $)_{2} \mathrm{ReO}_{4}$ salt and $t_{1}=194 \mathrm{meV}$, $t_{2}=158 \mathrm{meV}, U=1197 \mathrm{meV}$ and $W_{0}=100 \mathrm{meV}$ for [(TMTSF $\left.)_{0.5}(\text { TMTTF })_{0.5}\right]_{2} \mathrm{ReO}_{4}$ alloy [6,9], the charge gaps are evaluated as $\Delta_{\rho}=63.4 \mathrm{meV}$ for $(\mathrm{TMTTF})_{2} \mathrm{ReO}_{4}$ and $\Delta_{\rho}=198 \mathrm{meV}$ for $\left[(\mathrm{TMTSF})_{0.5}(\mathrm{TMTTF})_{0.5}\right]_{2} \mathrm{ReO}_{4}$, respectively. The comparison with the $\mathrm{RG}$ theoretical estimates [9] and the experimental data are shown in Table 1 as well.

In fact, above quantum self-consistent procedure is also equal to applying a Bogoliubov transformation [12] to the bosonized Hamiltonian. The condition of diagonalizing the quadratic terms leads to a same self-consistent equation for the transformation parameter. Moreover, such a Bogoliubov transformation can be regarded as a simple rescaling of the normal-mode coordinates and momenta, so that the elementary excitation of the model is also renormalized. 
Table 1

The parameters for the model and the charge gap from the experiments and the theoretical estimations for the Bechgaard salts

\begin{tabular}{|c|c|c|c|c|c|c|c|c|}
\hline & \multirow[t]{2}{*}{$t_{1}(\mathrm{meV})$} & \multirow[t]{2}{*}{$t_{2}(\mathrm{meV})$} & \multirow[t]{2}{*}{$U(\mathrm{meV})$} & \multirow[t]{2}{*}{$W_{0}(\mathrm{meV})$} & \multicolumn{4}{|c|}{$\Delta_{\rho}(\mathrm{meV})$} \\
\hline & & & & & Exp.[3] & DMRG [8] & RG [9] & Ours \\
\hline \multirow[t]{2}{*}{$(\mathrm{TMTTF})_{2} \mathrm{PF}_{6}$} & 130 & 90 & 910 & 0 & 100 & 35 & - & 64.4 \\
\hline & 137 & 93 & 959 & 0 & & - & 91 & 75.4 \\
\hline \multirow[t]{2}{*}{$(\mathrm{TMTSF})_{2} \mathrm{ClO}_{4}$} & 290 & 260 & 1450 & 0 & 25 & 6.5 & - & 11.5 \\
\hline & 287 & 266 & 1435 & 0 & & - & 7.5 & 6.21 \\
\hline$(\mathrm{TMTTF})_{2} \mathrm{R}_{2} \mathrm{O}_{4}$ & 140 & 100 & 959 & 0 & 65 & - & 76 & 63.4 \\
\hline$\left[(\mathrm{TMTSF})_{0.5}(\mathrm{TMTTF})_{0.5}\right]_{2} \mathrm{R}_{2} \mathrm{O}_{4}$ & 194 & 158 & 1197 & 100 & 95 & - & 270 & 198 \\
\hline
\end{tabular}

However, present theory is a fully quantum mechanical approach, and it systematically adds up the contributions of a large number of Feynman diagrams, with the loop number running from the lowest to infinite [12]. It, therefore, follows that our method, as an analytical approach, is superior to the RG one [9].

In summary we have applied an extended quantum selfconsistent method to the bosonization Hamiltonian to examine the charge gap in the $1 \mathrm{D}$ organic conductors at quarter filling which is described by the 1D Hubbard model with dimerization and alternate potential on site. In the weak coupling limit our results are in good agreement with those of the numerical and the other analytical theories. In the large and intermediate coupling regimes our results are comparable with that from the DMRG method. Our results are also consistent with the experimental data of the actual organic materials when the effect of nearest-neighbor Coulomb interaction is taken into account.

\section{Acknowledgements}

X.F.J. was supported in part by the Natural Science Foundation of Jiangsu Educational Department, China (Grant No. 03KJB140001). The authors are also grateful to the support from the NSC of Taiwan under Grant No. NSC91-2816-M-002-0009-6. We thank the referee for pointing out that Eq. (15) is exactly the same as that of SCHA.

\section{References}

[1] C. Coulon, P. Delhaes, S. Handrois, R. Lagnier, E. Bonjour, J.M. Fabre, J. Phys. France 43 (1982) 1059.

[2] D. Jérome, H.J. Schulz, Adv. Phys. 31 (1982) 229.

[3] V. Vescoli, L. Degiorgi, W. Henderson, G. Grüner, K.P. Sarkey, L.K. Montgomery, Science 281 (1998) 1181

[4] V.J. Emery, R. Bruinsma, S. Barisic, Phys. Rev. Lett. 48 (1982) 1039.

[5] J.P. Pouget, S. Ravy, J. Phys. I. France 6 (1996) 1501. K. Penc, F. Mila, J. Phys. IV Colloq. 3 (1993) C2-155.

[6] V. Ilakovac, et al., Phys. Rev. B 50 (1994) 7136.

[7] K. Penc, F. Mila, Phys. Rev. B 50 (1994) 11429.

[8] S. Nishimoto, M. Takahashi, Y. Ohta, J. Phys. Soc. Jpn 69 (2000) 1594.

[9] M. Tsuchiizu, H. Yoshioka, Y. Suzumura, J. Phys. Soc. Jpn 68 (1999) 1809.

[10] S. Nishimoto, Y. Ohta, Phys. Rev. B 59 (1999) 4738.

[11] T. Nakano, H. Fukuyama, J. Phys. Soc. Jpn 50 (1981) 2489.

[12] G.M. Zhang, H. Chen, X. Wu, Phys. Rev. B 43 (1991) 13566.

[13] Y.M. Zhang, B.W. Xu, W.F. Lu, Phys. Rev. B 49 (1994) 854.

[14] Eq. (2) can be also rewritten as the standard sine-Gordon formula by transforming the phase variable, seen from Ref. [9]. Here, for general, we do not do so.

[15] L. Ducasse, M. Abderrabba, J. Hoarau, M. Pesquer, B. Gallois, J. Gaultier, J. Phys. C 19 (1986) 3805.

[16] F. Mila, Phys. Rev. B 52 (1995) 4788. 